\title{
ON MOMENT CONDITIONS FOR SUPREMUM OF NORMED SUMS OF MARTINGALE DIFFERENCES
}

\author{
Wi Chong Ahn, Bong Dae Chol and Soo Hak Sung
}

Let $\left\{S_{n}, n \geqslant 1\right\}$ denote the partial sum of sequence $\left(X_{n}\right)$ of identically distributed martingale differences. It is shown that $E\left|X_{1}\right|^{q}\left(\lg \left|X_{1}\right|\right)^{r}<\infty$ implies $E\left(\sup \left((\lg n)^{p r / q} / n^{p / q}\right)\left|S_{n}\right|^{p}\right)<\infty$, where $1<p<2, p<q, r \in R$ and $\lg x=\max \left\{1, \log ^{+} x\right\}$ For the independent identically distributed case, the converse of the above statement holds.

\section{INTRODUCTION}

Let $\left\{X_{n}, n \geqslant 1\right\}$ be a sequence of random variables and $\left\{c_{n}, n \geqslant 1\right\}$ constants such that $0<c_{n} \uparrow \infty$. For each $n \geqslant 1$, let $S_{n}=X_{1}+\cdots+X_{n}$. In this paper, we will investigate the conditions on $\left(X_{n}\right)$ and $\left(c_{n}\right)$ under which

$$
E\left(\sup _{n}\left|S_{n}\right|^{p} / c_{n}\right)<\infty
$$

For independent identically distributed (i.i.d.) random variables $\left(X_{n}\right)$ with $E X_{1}=$ 0 and $c_{n}=n^{p / q}(1<q<2, p<q)$, it was shown by Choi and Sung [1] that (1.1) is equivalent to $E\left|X_{1}\right|^{q}<\infty$. This paper is a continuation of [1], and for the references about related works to the equivalent statements for (1.1), see [1].

In this paper, first we find conditions on $\left(c_{n}\right)$ to guarantee the statement (1.1) when $\left(X_{n}\right)$ is a sequence of identically distributed martingale differences. From this result, it is shown that if $\left(X_{n}\right)$ are independent identically distributed with $E X_{1}=0$ and $c_{n}=$ $n^{p / q} /(\lg n)^{p r / q}(1<q<2, p<q, r \in R)$ then (1.1) is equivalent to $E\left|X_{1}\right|^{q}\left(\lg \left|X_{1}\right|\right)^{r}<$ $\infty$, where $\lg x=\max \left\{1, \log ^{+} x\right\}$. When $r=0$, this equivalence is reduced to the one mentioned above.

Throughout this paper, $C>0$ will always stand for a constant which may be different in various places. $I(A)$ means the indicator function of event $A$.

Received 20th April 1990

This research was supported by Korea Science and Engineering Foundation 1989.

Copyright Clearance Centre, Inc. Serial-fee code: 0004-9729/91 \$A2.00+0.00. 


\section{Main results}

The following theorem [1] is essential for our main result and gives a sufficient condition of (1.1) for general increasing sequences $\left(c_{n}\right)$ and positive constants $\alpha(0<\alpha<2)$.

Theorem 1. Let $\left\{X_{n}, n \geqslant 1\right\}$ be a sequence of random variables and $\left\{c_{n}, n \geqslant\right.$ $1\}$ constants such that $0<c_{n} \uparrow \infty$. If $\sum_{n=1}^{\infty} E\left|X_{n}\right|^{\alpha \beta} / c_{n}^{\beta}<\infty$ for some $\beta>1$ and $0<\alpha \beta \leqslant 2$, then

$$
E\left(\sup _{n} \frac{\left|\sum_{k=1}^{n}\left(X_{k}-\alpha_{k}\right)\right|^{\alpha}}{c_{n}}\right)<\infty
$$

where $\alpha_{k}=0$ if $0<\alpha \beta \leqslant 1$ and $\alpha_{k}=E\left(X_{k} \mid X_{1}, \cdots, X_{k-1}\right)$ if $1<\alpha \beta \leqslant 2$.

The next result gives conditions on $\left(c_{n}\right)$ to guarantee the statement (1.1).

TheOREM 2. Let $\left\{X_{n}, n \geqslant 1\right\}$ be a sequence of identically distributed martingale differences and $\left\{c_{n}, n \geqslant 1\right\}$ constants such that $0<c_{n} \uparrow \infty$ and

$$
\sum_{n=1}^{\infty} P\left(\left|X_{1}\right|^{p}>c_{n}\right)<\infty
$$

If $c_{n}^{2 / p} \sum_{i=n}^{\infty} 1 / c_{i}^{2 / p}=O(n)$ and $c_{n}^{\beta} \sum_{i=1}^{n} 1 / c_{i}^{\beta}=O(n)$ for some $\beta$ with $1 \leqslant p \beta \leqslant 2$ and $1<\beta$, then

$$
E\left(\sup _{n} \frac{\left|S_{n}\right|^{p}}{c_{n}}\right)<\infty
$$

Proof: Define $\mathcal{F}_{n}=\sigma\left\{X_{1}, \cdots, X_{n}\right\}, Y_{n}=X_{n} I\left(\left|X_{n}\right|^{p} \leqslant c_{n}\right)$ $-E\left(X_{n} I\left(\left|X_{n}\right|^{p} \leqslant c_{n}\right) \mid \mathcal{F}_{n-1}\right)$ and $Z_{n}=X_{n} I\left(\left|X_{n}\right|^{p}>c_{n}\right)-E\left(X_{n} I\left(\left|X_{n}\right|^{p}>c_{n}\right) \mid \mathcal{F}_{n-1}\right)$. Then $X_{n}=Y_{n}+Z_{n}$. The proof will be completed by showing that

$$
E\left(\sup _{n} \frac{\left|\sum_{k=1}^{n} Y_{k}\right|^{p}}{c_{n}}\right)<\infty
$$

and

$$
E\left(\sup _{n} \frac{\left|\sum_{k=1}^{n} Z_{k}\right|^{p}}{c_{n}}\right)<\infty
$$

Result (2.1) is proved by applying Theorem 1 to the case $\alpha=p$ and $\beta=2 / p$, if we show that

$$
\sum_{n=1}^{\infty} \frac{E\left|Y_{n}\right|^{2}}{c_{n}^{2 / p}}<\infty
$$


Since $E\left|Y_{n}\right|^{2} \leqslant E\left|X_{1}\right|^{2} I\left(\left|X_{1}\right|^{p} \leqslant c_{n}\right)$, we have

$$
\begin{aligned}
\sum_{n=1}^{\infty} \frac{E\left|Y_{n}\right|^{2}}{c_{n}^{2 / p}} & \leqslant \sum_{n=1}^{\infty} \frac{1}{c_{n}^{2 / p}} E\left|X_{1}\right|^{2} I\left(\left|X_{1}\right|^{p} \leqslant c_{n}\right) \\
& =\sum_{n=1}^{\infty} \frac{1}{c_{n}^{2 / p}} \sum_{i=1}^{n} E\left|X_{1}\right|^{2} I\left(c_{i-1}<\left|X_{1}\right|^{p} \leqslant c_{i}\right) \quad\left(c_{0} \equiv 0\right) \\
& =\sum_{i=1}^{\infty} E\left|X_{1}\right|^{2} I\left(c_{i-1}<\left|X_{1}\right|^{p} \leqslant c_{i}\right) \sum_{n=i}^{\infty} \frac{1}{c_{n}^{2 / p}} \\
& \leqslant \sum_{i=1}^{\infty} P\left(c_{i-1}<\left|X_{1}\right|^{p} \leqslant c_{i}\right) c_{i}^{2 / p} \sum_{n=i}^{\infty} \frac{1}{c_{n}^{2 / p}} \\
& \leqslant C \sum_{i=1}^{\infty} P\left(c_{i-1}<\left|X_{1}\right|^{p} \leqslant c_{i}\right) i \\
& =C \sum_{i=0}^{\infty} P\left(\left|X_{1}\right|^{p}>c_{i}\right)<\infty .
\end{aligned}
$$

To prove (2.2), by Theorem 1, it is enough to show that

$$
\sum_{n=1}^{\infty} \frac{E\left|Z_{n}\right|^{p \beta}}{c_{n}^{\beta}}<\infty
$$

Since $E\left|Z_{n}\right|^{p \beta} \leqslant 2^{p \beta} E\left|X_{1}\right|^{p \beta} I\left(\left|X_{1}\right|^{p}>c_{n}\right)$, we have

$$
\begin{aligned}
\sum_{n=1}^{\infty} \frac{E\left|Z_{n}\right|^{p \beta}}{c_{n}^{\beta}} & \leqslant 2^{p \beta} \sum_{n=1}^{\infty} \frac{1}{c_{n}^{\beta}} E\left|X_{1}\right|^{p \beta} I\left(\left|X_{1}\right|^{p}>c_{n}\right) \\
& =2^{p \beta} \sum_{n=1}^{\infty} \frac{1}{c_{n}^{\beta}} \sum_{i=n}^{\infty} E\left|X_{1}\right|^{p \beta} I\left(c_{i}<\left|X_{1}\right|^{p} \leqslant c_{i+1}\right) \\
& =2^{p \beta} \sum_{i=1}^{\infty} E\left|X_{1}\right|^{p \beta} I\left(c_{i}<\left|X_{1}\right|^{p} \leqslant c_{i+1}\right) \sum_{n=1}^{i} \frac{1}{c_{n}^{\beta}} \\
& \leqslant 2^{p \beta} \sum_{i=1}^{\infty} P\left(c_{i}<\left|X_{1}\right|^{p} \leqslant c_{i+1}\right) c_{i+1}^{\beta} \sum_{n=1}^{i+1} \frac{1}{c_{n}^{\beta}} \\
& \leqslant C 2^{p \beta} \sum_{i=1}^{\infty} P\left(c_{i}<\left|X_{1}\right|^{p} \leqslant c_{i+1}\right)(i+1) \\
& \leqslant C 2^{p \beta} \sum_{i=0}^{\infty} P\left(\left|X_{1}\right|^{p}>c_{i}\right)<\infty .
\end{aligned}
$$


LEMMA 3. ([2], p.155) Let $X$ be a random variable and $\left\{c_{n}, n \geqslant 1\right\}$ constants such that $0<c_{n} \uparrow \infty$. Let $\phi$ be any even nondecreasing function satisfying $\phi\left(c_{n}\right)=n$ for all $n \geqslant 1$. Then

$$
E \phi(X)<\infty \text { if and only if } \sum_{n=1}^{\infty} P\left(|X|>c_{n}\right)<\infty .
$$

Let $\phi(x)=q^{r} x^{q}(\lg x)^{r}$ on $[0, \infty)$ for $1<q<2$ and $r \in R$. Since $\phi^{\prime}(x)$ is positive for large $x$ and $\phi(x) \rightarrow \infty$ as $x \rightarrow \infty$, we can choose an increasing sequence $\left(c_{n}\right)$ such that $\phi\left(c_{n}\right)=n$ for $n \geqslant n_{0}$ and $c_{n} \rightarrow \infty$. Thus we obtain a nondecreasing sequence $\left\{c_{n}, n \geqslant 1\right\}$ by letting $c_{n}=c_{n_{0}}$ for $1 \leqslant n<n_{0}$. Then we have $c_{n} \sim n^{1 / q} /(\lg n)^{r / q}$ by the following calculation: from the identity $\phi\left(c_{n}\right)=n$, that is, $q^{r} c_{n}^{q}\left(\lg c_{n}\right)^{r}=n$,

$$
\begin{aligned}
\frac{n^{1 / q}}{c_{n}(\lg n)^{r / q}} & =\frac{q^{r / q} c_{n}\left(\lg c_{n}\right)^{r / q}}{c_{n}\left(r \log q+q \lg c_{n}+r \lg \left(\lg c_{n}\right)\right)^{r / q}} \\
& =\frac{\left(q \lg c_{n}\right)^{r / q}}{\left(r \log q+q \lg c_{n}+r \lg \left(\lg c_{n}\right)\right)^{r / q}} \rightarrow 1 \text { as } n \rightarrow \infty
\end{aligned}
$$

Thus there exists an integer $\mathrm{N}$ such that

$$
1-\varepsilon<n^{1 / q} / c_{n}(\lg n)^{r / q}<1+\varepsilon
$$

for $n \geqslant N$. Hence we have

$$
\sum_{n=N}^{\infty} P\left(|X|>(1+\varepsilon) c_{n}\right)<\sum_{n=N}^{\infty} P\left(|X|>\frac{n^{1 / q}}{(\lg n)^{r / q}}\right)<\sum_{n=N}^{\infty} P\left(|X|>(1-\varepsilon) c_{n}\right) .
$$

Since $E|X|^{q}(\lg |X|)^{r}<\infty$ if and only if $E(C|X|)^{q}(\lg C|X|)^{r}<\infty$, we have by Lemma 3 that

$$
E|X|^{q}(\lg |X|)^{r}<\infty \text { if and only if } \sum_{n=1}^{\infty} P\left(|X|>C c_{n}\right)<\infty \text {. }
$$

Thus we have that

$$
E|X|^{q}(\lg |X|)^{r}<\infty \text { if and only if } \sum_{n=1}^{\infty} P\left(|X|>\frac{n^{1 / q}}{(\lg n)^{r / q}}\right)<\infty .
$$

THEOREM 4. Let $\left\{X_{n}, n \geqslant 1\right\}$ be a sequence of identically distributed martingale differences with $E\left|X_{1}\right|^{q}\left(\lg \left|X_{1}\right|\right)^{r}<\infty$ for $1<q<2$ and $r \in R$. Then for $p<q$

$$
E\left(\sup _{n}\left(\frac{(\lg n)^{r / q}}{n^{1 / q}}\right)^{p}\left|S_{n}\right|^{p}\right)<\infty
$$


ProOF: Let $c_{n}=\left(n^{1 / q} /(\lg n)^{r / q}\right)^{p}$. Choose a constant $\beta$ with $\beta=s / p(p<s<q, 1 \leqslant s<q)$. Then we have by $(2.4)$ that $\sum_{n=1}^{\infty} P\left(\left|X_{1}\right|^{p}>c_{n}\right)<\infty$. Some computation shows that

$$
\int_{1}^{n} \frac{(\lg x)^{s r / q}}{x^{s / q}} d x \leqslant C \frac{(\lg n)^{s r / q}}{n^{(8 / q)-1}}
$$

Thus we have

$$
c_{n}^{\beta} \sum_{i=1}^{n} \frac{1}{c_{i}^{\beta}} \leqslant C \frac{n^{s / q}}{(\lg n)^{\alpha / q}} \int_{1}^{n} \frac{(\lg x)^{e r / q}}{x^{s / q}} d x \leqslant C n .
$$

Similarly we have

$$
c_{n}^{2 / p} \sum_{i=n}^{\infty} \frac{1}{c_{i}^{2 / p}} \leqslant C \frac{n^{2 / q}}{(\lg n)^{2 r / q}} \int_{n}^{\infty} \frac{(\lg x)^{2 r / q}}{x^{2 / q}} d x \leqslant C n .
$$

Thus the result follows from Theorem 2 .

COROLlaRY 5. Let $\left\{X, X_{n}, n \geqslant 1\right\}$ be i.i.d. random variables with mean zero. Then the followings are equivalent: for $1<q<2, p<q$ and $r \in R$

$$
\begin{aligned}
& \text { (a) } E\left(\sup _{n}\left(\frac{(\lg n)^{r / q}}{n^{1 / q}}\right)^{p}\left|S_{n}\right|^{p}\right)<\infty ; \\
& \text { (b) } E\left(\sup _{n}\left(\frac{(\lg n)^{r / q}}{n^{1 / q}}\right)^{p}\left|X_{n}\right|^{p}\right)<\infty ; \\
& \text { (c) } E|X|^{q}(\lg |X|)^{r}<\infty .
\end{aligned}
$$

PROOF: The proof is similar to [1] and is omitted.

REMARK. The result in [1] is a special case of Corollary 5 in the case $r=0$.

\section{REFERENCES}

[1] B. D. Choi and S. H. Sung, 'On moment conditions for supremum of normed sums', Stochastic Process. Appl. 26 (1987), 99-106.

[2] W. F. Stout, Almost Sure Convergence (Academic Press, New York, 1974).

Department of Mathematics Education

Koomin University

Seoul 136-702

Korea

Coding Technology Section I

Electronics and Telecommunications

$$
\text { Research Institute }
$$

Taejeon 305-606

Korea
Department of Applied Mathematics

Korea Advanced Institute of Science and Technology

Seoul 130-650

Korea 